\title{
Injury to Urachal Diverticulum Due to Laparoscopy Port: A Case Report, Literature Review and Recommendations
}

\author{
Kaushik Kumara, b, Daniel Mehanna ${ }^{\mathrm{a}}$
}

\begin{abstract}
Minimally invasive surgery has gained widespread acceptance in elective and emergency situations due to reduced pain, length of hospital stay for patients and wider view of the operative field. However, it does bring its own set of specific risks and complications that must be kept in mind. Injury to the urinary bladder, in particular in the setting of an undiagnosed urachal abnormality can easily go unrecognized at the time of operation. Injury to an unsuspected urachal diverticulum due to laparoscopic port placement is an extremely rare complication due to the very low incidence of this abnormality. We report a case of a 10-year-old boy who sustained an injury to an urachal diverticulum during a routine laparoscopic appendicectomy and give recommendations to minimize the risk of injury to the urinary bladder or an associated urachal diverticulum.
\end{abstract}

Keywords: Urachus; Bladder diverticulum; Laparoscopy; Urinary bladder

\section{Introduction}

Laparoscopic surgeries are gaining popularities in all surgical fields in acute and elective settings. It brings its own specific complications related to entry and port placement of laparoscopy. Accessory ports placement in suprapubic region while doing appendicectomy or diagnostic laparoscopy can cause injury to urinary bladder or urachal remnants when present. Many times it is junior registrar or trainee who is performing unsupervised procedures. All surgeons should have high index of suspicion for this rare entity as unrecognized injuries can cause diagnostic confusion in post-operative period and may have higher morbidity. We reported a case of injury to

Manuscript accepted for publication November 14, 2014

${ }^{a}$ General Surgery Department, Caboolture Private Hospital, Caboolture, QLD 4510, Australia

${ }^{\mathrm{b}}$ Corresponding Author: Kaushik Kumar, 3, Kennedy Court, Urraween, QLD 4655, Australia. Email: drkaushik1977@gmail.com

doi: http://dx.doi.org/10.14740/jmc2000w bladder due to urachal diverticulum due to laparoscopic port, performed literature review for similar cases and suggested methods for diagnosis and prevention of such injuries.

\section{Case Report}

A previously well 10-year-old boy underwent a laparoscopic appendectomy for ultrasound proven appendicitis. A 10-mm infra-umbilical port was inserted by an open technique and pneumoperitoneum created without difficulty. Two additional $5-\mathrm{mm}$ ports were also placed under vision in the suprapubic and left abdominal regions. In retrospect, it was noted that although the suprapubic port was placed high to avoid the bladder, there was some difficulty in the placement and counter pressure of the abdominal wall with laparoscopic bowel graspers was needed to facilitate placement. An inflamed appendix was noted with free fluid in the pelvis and a routine appendicectomy was performed. A 10-F Blake drain was placed via the suprapubic port and the patient was admitted overnight for observation. Upon review in the morning, the drain output was noted to be nearly $2,000 \mathrm{~mL}$ of serous fluid. His abdominal pain was only mild and observations were normal apart from decreased total urine output since surgery. On examination, his abdomen was soft with mild lower abdominal tenderness without any signs of peritonism.

Due to history of difficulty in placement of suprapubic port, unusual amount of drain output and decreased total urine output, an injury to urinary bladder was suspected. An indwelling urinary catheter was placed and a CT cystogram was performed using $200 \mathrm{~mL}$ of dilute water soluble contrast. The scan showed a midline vesicourachal diverticulum extending superiorly to just below the umbilicus. The drain, which had been placed via the suprapubic port, was noted to be passing directly through the vesicourachal diverticulum with contrast leaking around the drain and into the pelvis (Fig. 1,2). With the assistance of an urologist, the patient was taken back to theater for a re-laparoscopy. At operation, the drain was removed under vision and a small $5-\mathrm{mm}$ defect was identified in the bladder. The injury was repaired laparoscopically with a two-layered vicryl closure. A drain was placed via the left-sided abdominal port. Post-operatively, the patient recovered well and was discharged on the third postoperative day with an indwelling urinary catheter and leg bag. The catheter was subsequently 


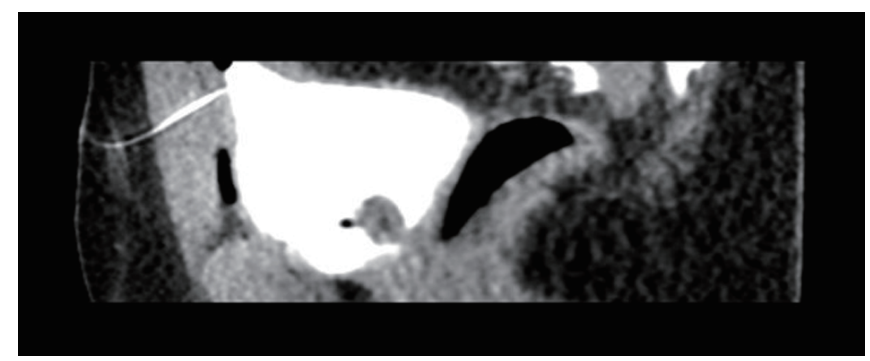

Figure 1. CT cystogram showing urachal diverticular and suprapubic drain can be seen passing through diverticulum.

removed 10 days later following a normal cystogram.

\section{Discussion}

Minimally invasive surgery has gained widespread acceptance in elective and emergency situations due to reduced pain, length of hospital stay for patients and wider view of the operative field. Laparoscopic appendectomy is one of the most commonly performed emergency procedures and is usually performed with minimal morbidity. Diagnostic laparoscopy has also gained popularity due to its ability to supersede radiological imaging in terms of diagnostic specificity and to facilitate treatment in the same sitting. However, the laparoscopic approach does bring its own specific complications including bleeding, omental injury and injuries to hollow organs such as bowel, bladder or ureters. The urinary bladder is particularly vulnerable to injury by port placement in the suprapubic region due to its location, distensibility and thin wall [1].

\section{Method of literature review}

A PubMed search was conducted based on the keywords: "Urachus", "Urinary bladder diverticulum", "Urinary bladder injury" and "Laparoscopic port".

\section{Complication rate of laparoscopy}

The overall rate of major complications following a laparoscopic procedure is approximately 1.4 per 1,000 cases. This includes gastrointestinal ( 0.6 per 1,000 cases), urological $(0.3$ per 1,000 cases), vascular ( 0.1 per 1,000 cases) and omental injuries $(0.4 \%)$ [2]. The incidence of injury to the urinary blad-

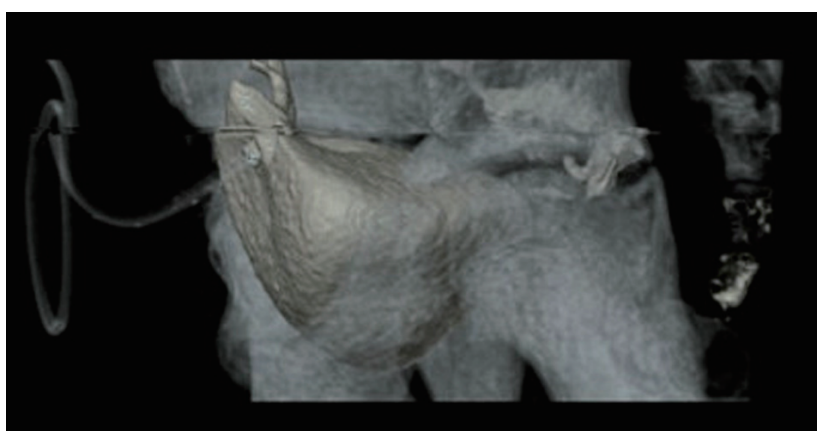

Figure 2. The reconstructed image of CT cystogram showing urachal diverticulum and position of drain.

der varies between $0.2 \%$ (diagnostic laparoscopies) and up to $8 \%$ (operative laparoscopies) [1,3]. Injury to the urinary bladder due to suprapubic port insertion is rare. Levy et al reported six cases of bladder injury due to suprapubic port placement in 1,671 patients (incidence 0.36\%) [4], with one of the six patients having an urachal diverticulum.

\section{Urachal abnormalities}

The urachus is a tubular structure that drains the urinary bladder of the foetus and joins the allantois at the umbilicus $[5,6]$. The bladder develops from the ventral cloaca and is connected with the allantois at its upper end. Between the fourth and fifth months of gestation, the allantois regresses and the urachus obliterates completely to become a fibromuscular strand known as median umbilical ligament. After birth, the urinary bladder is mostly an intraperitoneal organ, while the urachus remains in the extraperitoneal space of Retzius (the space between the transversalis fascia and peritoneum). Histologically, it is composed of all the three bladder layers, namely mucosa, lamina propria and muscular layer.

Failure of obliteration of urachus results in urachal abnormalities, of which there are four types (Fig. 3). 1) Patent urachus: the lumen of the urachus remains patent from the bladder to the umbilicus and it may discharge urine from the umbilicus. 2) Urachal sinus: only the distal part of tract involutes, while the proximal part remains patent and attached to the umbilicus. This can be injured during insertion of the umbilical port at laparoscopy [7]. 3) Vesicourachal diverticulum: the distal part remains patent and connected with bladder, while proximal part obliterates. 4) Urachal cyst: obliteration of tract occurs on both ends, but remains patent in between at one or

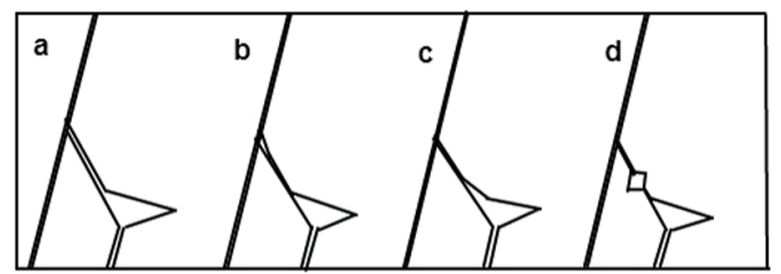

Figure 3. Types of urachal anomalies: (a) patent urachus; (b) urachal sinus; (c) urachal diverticulum; (d) urachal cyst. 
Table 1. Classification of latrogenic Injuries to the Urinary Bladder

\begin{tabular}{ll}
\hline Grade 1 & Contusion, intramural hematoma or partial thickness laceration \\
Grade 2 & Extraperitoneal bladder wall laceration $<2 \mathrm{~cm}$ \\
Grade 3 & Extraperitoneal bladder wall laceration $>2 \mathrm{~cm}$ or intraperitoneal bladder wall laceration $<2 \mathrm{~cm}$ \\
Grade 4 & Intraperitoneal bladder wall laceration $>2 \mathrm{~cm}$ \\
Grade 5 & Intra- or extraperitoneal bladder wall laceration involving the trigone or bladder neck \\
\hline
\end{tabular}

more places, mostly near bladder.

The reported incidence of a patent urachus is 3 per 100,000 births $[2,4]$. Urachal abnormalities are very rarely diagnosed clinically but due to the increasing use of diagnostic modalities such as ultrasound and computer-assisted tomography, it is being recognized more frequently. Common complications associated with urachal remnants include pain, urinary infection, urachal abscess and stone formation. Cases of spontaneous perforation of urachal diverticulum or cysts $[8,9]$, diverticular tumors [10], urachal-sigmoid fistula [11], cholecysto-urachal fistula [12] and injury during laparoscopy have been described.

Port site injuries to urachal remnants are extremely rare and a review of the literature showed only six cases reported to date. One case involved injury to an urachal cyst [13], one to a possible patent urachus [7] and four cases involved injury to an urachal diverticulum $[1,4,5,14]$. Our case is the fifth such reported case of injury to an urachal diverticulum due to laparoscopic port insertion. It is interesting to note that even if the bladder is empty, it is still susceptible to damage on insertion of a suprapubic port as the diverticulum is situated in the space of Retzius.

\section{Urinary bladder injury: diagnosis and electrolyte distur- bances}

The key to the diagnosis of bladder injury is a high index of suspicion on the part of the surgeon [8]. Intra-operatively, it can be diagnosed by leakage of urine from the port insertion site or gas or blood in the urinary catheter. Ostrzenski and Ostrzenska in their review of the literature concluded that the recognition of a bladder injury was made intra-operatively in only $53.24 \%$ of cases [3]. Intra-operative detection can be aided by obviously visible bladder rent, urine leak or gaseous distension of urinary bag as noted in case reported by Classi [15]. If bladder injury is not recognized during surgery, it can lead to diagnostic uncertainty post-operatively as the resulting urinary peritonitis may lack specific symptoms and signs and can produce biochemical changes mimicking acute renal failure $[1$, 16].

Gascon et al [1] mentioned few findings in post-operative period which can be indicative of bladder injury: 1) persistent, irritating abdominal pain due to urinary peritonitis; 2) supposed anuria or low urine output that improves after insertion of an indwelling catheter; 3) intermittent hematuria; 4) elevated white cell count and profound disturbances in the serum electrolytes and acid base status without patient being febrile and features of infectious peritonitis. The elevated level of serum urea, creatinine and potassium, decreased level of sodium and $\mathrm{CO}_{2}$ and development of metabolic acidosis as consistent findings among patients with intraperitoneal rupture of urinary bladder due to reverse autodialysis produce a picture of pseudo renal failure [16].

Other important features that may suggest the possibility of a urinary tract injury include the presence of significant abdominal free fluid on imaging, the abnormal location of the urinary catheter bulb in imaging and unexpected high abdominal drain output in an otherwise straight forward procedure as was the case with our patient.

If suspected during surgery, the diagnosis can be confirmed by the instillation of methylene blue through the urinary catheter and confirmation of leak of dye intraperitoneally. This is simple and very useful test. Post-operatively, the diagnosis may be possible by ultrasound which may show an extravesical position of bulb of the urinary catheter as was reported by Gascon et al [1]. CT cystogram with retrograde instillation of water soluble contrast will show leak of contrast from the bladder and will also show any urachal remnant of the bladder as was observed in our case. The cystoscopy may be useful to evaluate the extent of bladder trauma in relation to the ureteric orifices. In suspected cases with unexpected high output from an intraabdominal drain, comparing drain fluid creatinine to serum creatinine may also be useful. If drain creatinine is equal to serum creatinine, a bladder injury is unlikely [17]. However, care must be taken in comparing serum, drainage and urine biochemical values because different measurement units may be used by laboratory for each.

\section{Management of urinary bladder injuries due to laparo- scopic port}

Management of bladder injuries depends on the size, site of the injury and how it is diagnosed. If diagnosed intraoperatively, it should be repaired at the time. Post-operatively diagnosed injuries can be managed with a wide range of techniques. Grade 2 urinary injuries can be managed with prolonged urinary catheterization, while grade $3-5$ injuries should be operatively repaired [17] (Table 1).

No definite recommendations exist for incidentally identified urachal remnants. Remnants with epithelium are at risk of development of carcinoma while those with fibrostromal tissue without epithelium may have a lower malignant potential [18]. In the cases of injury to urachal diverticula due to port placement, differing management strategies have been used in reported cases, including insertion of an indwelling catheterization of the bladder [4], suture repair [1,5] and excision of the diverticulum with closure of bladder defect [14]. The key 


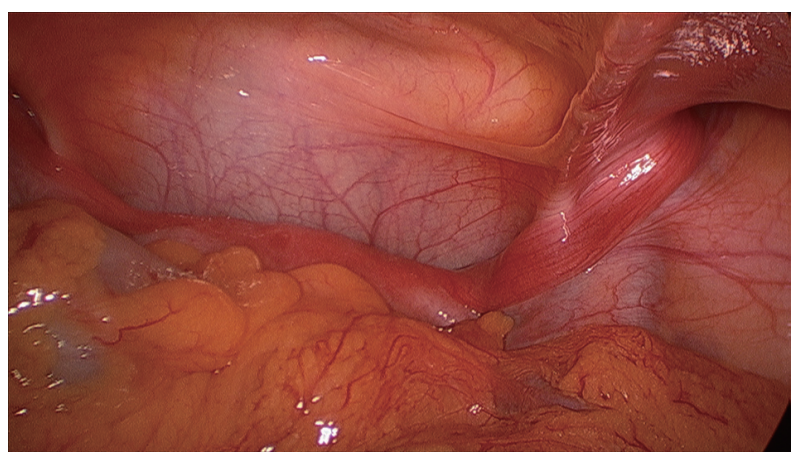

Figure 4. Laparoscopic view of superior border of urinary bladder which appears glistening white.

to successful management is early recognition of injury. In our case, re-laparoscopy was undertaken within $24 \mathrm{~h}$ and the drain was removed under vision and bladder injury repaired with sutures. A drain was then inserted via another port site.

\section{Prevention of bladder/urachal remnant injury during laparoscopic surgery}

The clinician should be aware of this rare anatomical variant and care should be taken when inserting a suprapubic port. Our recommendations include: 1) Pre-operative voiding or in-out catheterization. 2) Careful placement of the suprapubic port. While is it advantageous from a cosmetic perspective to have a low scar in pubic hair area, the port must not be placed too low. 3) In the event of difficulty in the insertion of the suprapubic port, the possibility of bladder or urachal diverticulum intervening must be considered. 4) Instillation of methylene blue via urethral catheter to exclude an injury if suspected at the time of initial surgery. Instillation of methylene blue into the indwelling catheter is a quick, safe and easily performed maneuvre which can be performed by a junior surgical registrar, who may be operating without direct supervision in the middle of the night. 5) Identification of the superior border of the bladder. The peritoneum over urinary bladder appears glistening white (Fig. 4) compared to the peritoneum above urinary bladder and it will give idea about upper border of urinary border and port can be safely placed under vision above that border. However, it may be difficult to identify it in cases of previous pelvic surgery, adhesions and urahcal diverticulum and in such cases alternate site port placement should be considered if possible. 6) All laparoscopic ports should be removed under vision and port sites should be inspected carefully for any bleeding or urine leak before completing the procedure. 7) In any post-operative patient with unexpected pain or distension, high unexpected drain output, reduced urinary output or anuria and typical blood chemistry, an injury to urinary bladder should be kept in mind.

\section{Conclusion}

Although having obvious advantages over tradition open sur- gery, laparoscopic surgery does bring its own set of specific risks and complications. Injury to the urinary bladder, in particular in the setting of an undiagnosed urachal abnormality can easily go unrecognized at the time of operation. Surgeons should take great care on port placement and must have a high index of suspicion in order to recognize a bladder injury. If the suprapubic port is particularly difficult to place then a bladder injury should be suspected and excluded intraoperatively with the instillation on methylene blue into the indwelling catheter. Positively identifying upper bladder boundary under direct vision will help to reduce the risk of injury to the bladder. In the event of unusual difficulty or failure to identify bladder boundary, alternate site port placement should be considered.

\section{Grant Support}

None.

\section{Conflict of Interest}

Authors declare no conflict of interest regarding this article.

\section{Author Contributions}

Dr Mehanna was the main surgeon who performed surgery and Dr Kumar assisted him. Patient's data, literature review and manuscript preparation were done by Dr Kumar. Both authors have reviewed and approved final version of manuscript.

\section{References}

1. Jacqueline G, Maribel A, Pedro A. Urinary peritonitis due to unrecognized bladder injury during laparoscopy: A case report. World J Nephrol Urol. 2013;2(1):21-24.

2. Krishnakumar S, Tambe P. Entry complications in laparoscopic surgery. J Gynecol Endosc Surg. 2009;1(1):4-11.

3. Ostrzenski A, Ostrzenska KM. Bladder injury during laparoscopic surgery. Obstet Gynecol Surv. 1998;53(3):175180.

4. Levy BF, De Guara J, Willson PD, Soon Y, Kent A, Rockall TA. Bladder injuries in emergency/expedited laparoscopic surgery in the absence of previous surgery: a case series. Ann R Coll Surg Engl. 2012;94(3):e118-120.

5. Nimmonrat A, Na-ChiangMai W, Muttarak M. Urachal abnormalities: clinical and imaging features. Singapore Med J. 2008;49(11):930-935.

6. Schnyder P, Candardjis G. Vesicourachal diverticulum: CT diagnosis in two adults. AJR Am J Roentgenol. 1981;137(5):1063-1065.

7. McLucas B, March C. Urachal sinus perforation during laparoscopy. A case report. J Reprod Med. 1990;35(5):573574.

8. Ohgaki M, Higuchi A, Chou H, Takashina K, Kawakami S, Fujita Y, Hagiwara A, et al. Acute peritonitis caused by 
intraperitoneal rupture of an infected urachal cyst: report of a case. Surg Today. 2003;33(1):75-77.

9. Ogbevoen JO, Jaffe DM, Langer JC. Intraperitoneal rupture of an infected urachal cyst: a rare cause of peritonitis in children. Pediatr Emerg Care. 1996;12(1):41-43.

10. Naiditch JA, Radhakrishnan J, Chin AC. Current diagnosis and management of urachal remnants. J Pediatr Surg. 2013;48(10):2148-2152.

11. Gomez Barbadillo J, Plata Rosales J, Espinosa Guzman E, Castilla Cabezas J, Diaz Lopez C, Soria Alvarez C, Ramos Cejudo F, et al. Urachal-sigmoid fistula in an adult male without urachal cyst. Rev Esp Enferm Dig. 2002;94(7):430-434.

12. Rabinowitz CB, Song JH, Movson JS, Iannotti HM. Cholecysto-urachal fistula. Abdom Imaging. 2007;32(1):108110 .

13. Arenas-Marquez H, Hernandez-Zuniga JF, BarreraZepeda LM, Flores-Marquez MR, Chavez-Perez R. [Chain of errors in laparoscopic appendectomy]. Cir Cir.
2012;80(4):379-384.

14. Yong EL, Prabhakaran K, Lee YS, Ratnam SS. Peritonitis following diagnostic laparoscopy due to injury to a vesicourachal diverticulum. Case report. British J of Obstetrics and Gynaecology. 1989; 96:365-368.

15. Classi R, Sloan PA. Intraoperative detection of laparoscopic bladder injury. Can J Anaesth. 1995;42(5 Pt 1):415-416.

16. Vilos GA, Haebe J, Crumley TL, Maruncic MA, King JH, Denstedt JD. Serum biochemical changes after laparoscopy may be indicators of bladder injury. J Am Assoc Gynecol Laparosc. 2001;8(2):285-290.

17. Delacroix SE, Jr., Winters JC. Urinary tract injures: recognition and management. Clin Colon Rectal Surg. 2010;23(2):104-112.

18. Copp HL, Wong IY, Krishnan C, Malhotra S, Kennedy WA. Clinical presentation and urachal remnant pathology: implications for treatment. J Urol. 2009;182(4 Suppl):1921-1924. 